\title{
Magneto-controlled Quantized Electron Transfer to Surface-confined Redox Units and Metal Nanoparticles
}

\author{
Eugenii Katz ${ }^{*}$ and Itamar Willner \\ Institute of Chemistry, The Hebrew University of Jerusalem, Jerusalem 91940, Israel \\ *Author to whom correspondence should be addressed. E-mail: ekatz@vms.huji.ac.il
}

Received: 1 August 2005 / Accepted: 4 January 2006 / Published: 7 April 2006

\begin{abstract}
Hydrophobic magnetic nanoparticles (NPs) consisting of undecanoate-capped magnetite $\left(\mathrm{Fe}_{3} \mathrm{O}_{4}\right.$, average diameter ca. $\left.5 \mathrm{~nm}\right)$ are used to control quantized electron transfer to surface-confined redox units and metal NPs. A two-phase system consisting of an aqueous electrolyte solution and a toluene phase that includes the suspended undecanoatecapped magnetic NPs is used to control the interfacial properties of the electrode surface. The attracted magnetic NPs form a hydrophobic layer on the electrode surface resulting in the change of the mechanisms of the surface-confined electrochemical processes. A quinone-monolayer modified $\mathrm{Au}$ electrode demonstrates an aqueous-type of the electrochemical process $\left(2 \mathrm{e}^{-}+2 \mathrm{H}^{+}\right.$redox mechanism) for the quinone units in the absence of the hydrophobic magnetic NPs, while the attraction of the magnetic NPs to the surface results in the stepwise single-electron transfer mechanism characteristic of a dry nonaqueous medium. Also, the attraction of the hydrophobic magnetic NPs to the Au electrode surface modified with Au NPs (ca. $1.4 \mathrm{~nm}$ ) yields a microenvironment with a low dielectric constant that results in the single-electron quantum charging of the Au NPs.
\end{abstract}

Keywords: Nanoparticles, magnetic particles, quantum charging, modified electrode, switchable interface. 


\section{Introduction}

Chemically functionalized nanoparticles (NPs) can be associated with modified electrode surfaces and used to enhance electrochemical, photoelectrochemical or bioelectrochemical processes [1,2]. Functionalized magnetic NPs were used for separation of bioaffinity complexes [3,4] and for the construction of magnetic nanostructures [5]. Also, the functionalized magnetic NPs or microparticles were used for labeling of biomaterials, such as oligonucleotides or antigens/antibodies, that allowed designing new DNA-sensors [6,7] and immunosensors [8,9]. Recently, functionalized magnetic particles were employed for the ON-OFF switching of bioelectrocatalytic processes [10,11]. Also, the rotation of functionalized magnetic particles on electrode supports was used to amplify bioelectrocatalytic reactions [12,13] and biosensing processes such as DNA or antigen-antibody interactions [14,15] and cancer cells analysis [16]. Recently, we reported on the reversible blocking of the electrochemical and bioelectrocatalytic processes at electrodes by attraction of the hydrophobic magnetic NPs to the electrode surface $[17,18]$. The magnetic NPs (average diameter $5 \mathrm{~nm}$ ) modified with long alkyl chains form, upon their attraction to the electrode, a hydrophobic thin film on the conductive support. This thin film results in a low capacitance of the double-charged layer and complete blocking of diffusional electrochemical processes. Magnetic retraction of the hydrophobic magnetic NPs regenerates a bare electrode surface, which allows the duffusional electrochemical process. Reversible attraction/retraction of the hydrophobic magnetic NPs to/from the electrode surface provides a means to reversibly deactivate/activate the duffusional electrochemical processes. In the present study we report on the effect of hydrophobic magnetic NPs attracted to the electrode, by means of an external magnet, on the mechanisms of electrochemical processes at monolayerfunctionalized electrodes, namely on the quantized electron transfer to surface-confined quinone units and Au NPs.

\section{Experimental section}

\section{Chemicals and materials}

Undecanoic acid, cystamine (2,2'-diaminodiethyldisulfide), 2,3-dichloro-1,4-naphthoquinone, 4-(2hydroxyethyl)piperazine-1-ethanesulfonic acid sodium salt (HEPES), 1-ethyl-3-(3dimethylaminopropyl)-carbodiimide (EDC), and all other chemicals were purchased from Sigma or Aldrich and used without further purification. Monoamino-functionalized Au-nanoparticles (ca. $1.4 \mathrm{~nm}$ ) containing a single triphenylphosphine ligand functionalized with 1,3-propane diamine were purchased from Nanoprobes (U.S.A.). Magnetic nanoparticles, $\mathrm{Fe}_{3} \mathrm{O}_{4}$, coated with undecanoic acid shell were synthesized according to the published procedure with the difference that only a single capping layer was generated on the surface of the nanoparticles [19]. Ultrapure water from NANOpure Diamond $^{\mathrm{TM}}$ (Barnstead) source was used throughout all the experiments.

\section{Chemical modification of electrodes}

A Au-coated (50 nm gold layer) glass plate (Analytical $\mu$-Systems, Germany) was used as a working electrode. Cystamine was self-assembled on the electrode as a monolayer to yield the aminofunctionalized $\mathrm{Au}$ surface as described before [20,21]. The resulting amino-functionalized Au 
electrode was reacted with 2,3-dichloro-1,4-naphthoquinone in boiling ethanolic solution for 3 minutes and thoroughly rinsed with ethanol and water to yield the amino-naphthoquinone monolayer (1) at a modified electrode as described before [21]. 11-Mercaptoundecanoic acid (1 mM ethanolic solution) was self-assembled on the Au electrode overnight to yield the carboxylic-functionalized Au surface. The resulting modified Au electrode was then reacted overnight with the amino-functionalized Au NPs $\left(1 \mu \mathrm{g} \mathrm{mL}^{-1}\right)$ in $0.1 \mathrm{M}$ HEPES-buffer, $\mathrm{pH}=7.2$, in the presence of EDC, $10 \mathrm{mM}$, to yield the Au NPsfunctionalized interface.

Electrochemical and microgravimetric measurements

Cyclic voltammetry and linear sweep voltammetry measurements were performed using an electrochemical analyzer (model 6310, EG\&G connected to a personal computer with EG\&G 270/250 software). The measurements were carried out at ambient temperature $\left(25 \pm 2^{\circ} \mathrm{C}\right)$ in a conventional electrochemical cell consisting of a modified Au working electrode assembled at the bottom of the electrochemical cell, a glassy carbon auxiliary electrode and a saturated calomel electrode (SCE) connected to the working volume with a Luggin capillary. All potentials are reported with respect to this reference electrode. Phosphate buffer $(0.1 \mathrm{M}, \mathrm{pH} 7.0)$ was used as a background electrolyte for the electrochemical measurements. The undecanoic acid-functionalized magnetic NPs were added to the cell in a toluene solution $\left(0.5 \mathrm{~mL}, 1 \mathrm{mg} \mathrm{mL}^{-1}\right)$ yielding an upper organic solution layer immiscible with the aqueous electrolyte solution. The undecanoic acid-functionalized magnetic NPs were attracted to the modified Au electrode surface from the upper organic layer by positioning a $12 \mathrm{~mm}$ diameter magnet (NdFeB/Zn-coated magnet with the remanent magnetization of $10.8 \mathrm{kG}$ ) below the bottom electrode. The hydrophobic magnetic NPs were removed from the electrode surface and re-transported to the organic phase by positioning the external magnet on the top of the electrochemical cell. Argon bubbling was used to remove oxygen from the solutions in the electrochemical cell. The cell was placed in a grounded Faraday cage. A QCM analyzer (Fluke 164T multifunction counter, $1.3 \mathrm{GHz}$, TCXO) and quartz crystals (AT-cut, $9 \mathrm{MHz}$, Seiko) sandwiched between two Au electrodes (area $0.196 \mathrm{~cm}^{2}$, roughness factor ca. 3.2) were employed for the microgravimetric analyses in air.

\section{Results and discussion}

The magnetic NPs were prepared according to the literature procedure using undecanoic acid as a hydrophobic capping layer [19]. The undecanoic acid-functionalized NPs are freely suspendable in organic phases, such as toluene, and form a stable homogenous suspension (a magnetic fluid [22,23]). TEM and AFM images of the NPs indicated that the average diameter of the magnetic NPs is ca. $5 \mathrm{~nm}$ [17]. The saturation magnetization of the NPs at room temperature was determined to be ca. 36.4 emu $\mathrm{g}^{-1}[17]$.

The deposition of the magnetic NPs on the electrode surface as a thin film turns the interface into a hydrophobic medium that blocks diffusional electrochemical reactions at the electrode surface [18]. Surface-confined redox species should be still accessible to the electrical contact, and thus, their electrochemical response can be observed in the presence of the magnetic NPs on the electrode surface. However, the electrochemical processes on the functionalized interfaces in the presence of the hydrophobic magnetic NPs occur in the dry non-aqueous microenvironment generated by the thin film 
of the magnetic NPs. Thus, the magneto-controlled deposition of the hydrophobic NPs on modified electrode surfaces could, in principle, result in the significant change of the mechanisms of the electrochemical processes.

This concept was experimentally proved by studying the electrochemical processes of the quinone (1) monolayer-functionalized electrode in the absence and in the presence of the hydrophobic magnetic NPs on the electrode surface, Figures 1(A) and 1(B), respectively. The cyclic voltammogram of the system with the hydrophobic magnetic NPs retracted from the electrode surface is depicted in Figure $1(\mathrm{C})$, curve (a). It consists of a redox wave at $\mathrm{E}^{\circ}=-0.41 \mathrm{~V}$ corresponding to the reversible reduction of the quinone monolayer (1), $2.2 \times 10^{-11}$ mole $\mathrm{cm}^{-2}$. The surface-confined character of the electrochemical reaction corresponding to the quinone units is supported by the linear increase of the peak currents with the increase of the potential scan rate. When the hydrophobic magnetic NPs are attracted to the electrode surface, the cyclic voltammogram shows, however, two waves corresponding to the electrochemical process of the immobilized quinone (1), $\mathrm{E}_{1}{ }^{\circ}=-0.465 \mathrm{~V} ; \mathrm{E}_{2}{ }^{\circ}=-0.630 \mathrm{~V}$, Figure $1(\mathrm{C})$, curve (b). Also, a significant decrease in the capacitance current is observed under these conditions. The peak currents of the quinone reduction waves increase linearly with the increased potential scan rate indicating that the electron transfer occurs on a surface-confined species.

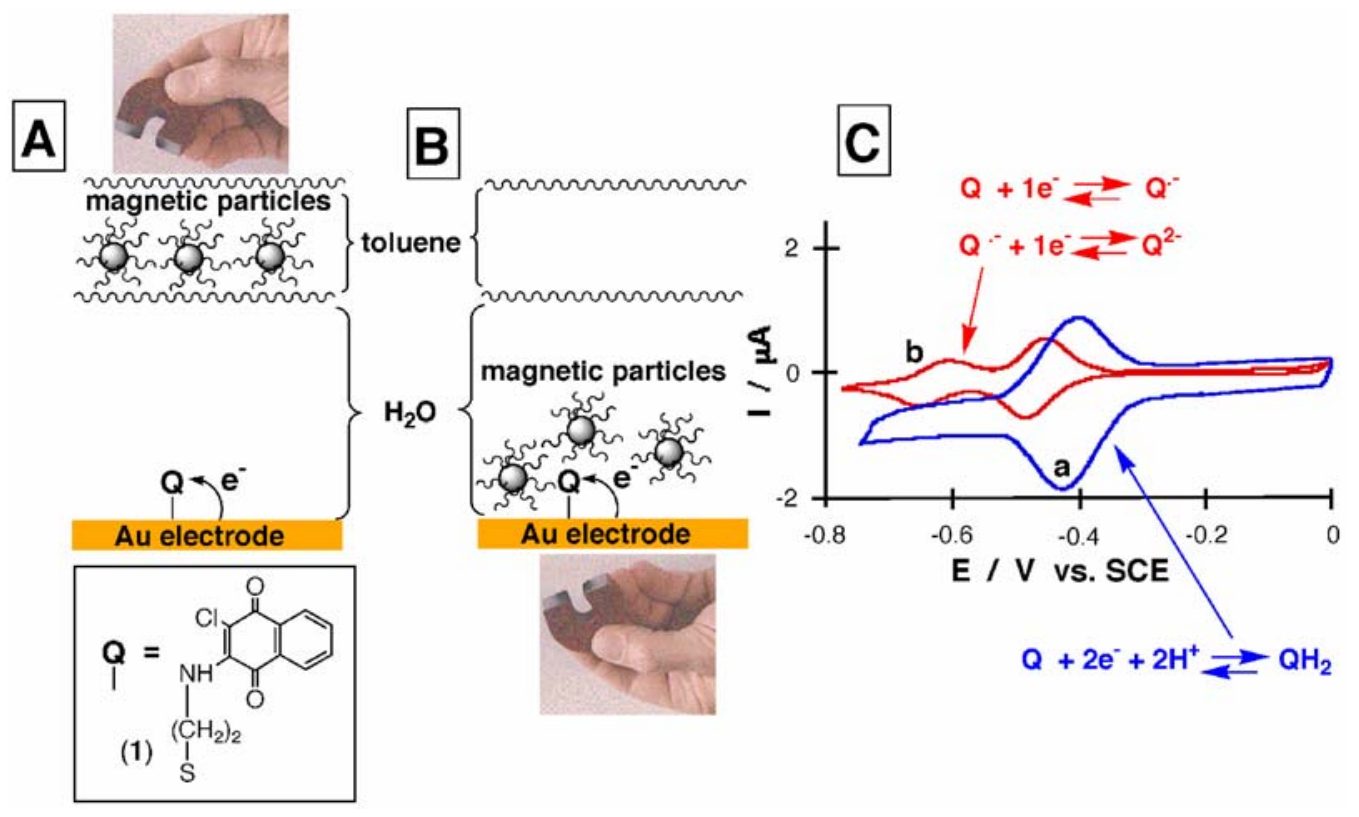

Figure 1. Magneto-controlled reversible translocation of the hydrophobic magnetic NPs between an organic phase, atop the aqueous electrolyte, and the electrode surface: (A) The magnetic NPs are retracted from the electrode surface and the electrode is activated towards the aqueous-type electrochemistry of the surface-confined quinone (1). (B) The electrode surface is blocked by the hydrophobic magnetic NPs attracted to the electrode by the external magnet, while the surfaceconfined quinone (1) reveals the non-aqueous type electrochemistry. (C) Cyclic voltammograms of the system consisting of the surface-confined quinone (1): a) When the hydrophobic magnetic NPs are retracted from the electrode surface. b) When the hydrophobic magnetic NPs are attracted to the electrode surface. Data were recorded at room temperate under Ar in a biphase system composed of $0.1 \mathrm{M}$ phosphate buffer, $\mathrm{pH} 7.0$ (lower phase) and toluene with the magnetic NPs, $0.5 \mathrm{~mL}, 1 \mathrm{mg} \mathrm{mL}^{-1}$, (upper phase). The effective electrode area is ca. $0.3 \mathrm{~cm}^{2}$. Potential scan rate is $100 \mathrm{mV} \mathrm{s}^{-1}$. 
It is well known that in an aqueous medium the electrochemistry of quinones shows a single redox wave corresponding to the $\left(2 \mathrm{e}^{-}+2 \mathrm{H}^{+}\right)$-electrochemical reduction, whereas in dry non-aqueous solutions (e.g., in acetonitril, dichloromethane, or dimethylformamide) quinones show two consecutive redox waves corresponding to the (1 $\left.\mathrm{e}^{-}\right)$-reduction steps each [24]. The addition of water to non-aqueous solvents (sometimes traces of water in non-aqueous solvents) results in the protonation of anionic reduced states of quinones and it changes the electrochemical process of quinones, typical to dry nonaqueous environment to the mechanism characteristic for aqueous solutions [24]. The two almost equal redox waves corresponding to the electrochemical process of the immobilized quinone (1), $\mathrm{E}_{1}{ }^{\circ}=$ $-0.465 \mathrm{~V} ; \mathrm{E}_{2}{ }^{\circ}=-0.630 \mathrm{~V}$, demonstrate that the attraction of the hydrophobic magnetic NPs to the electrode surface provides a non-aqueous medium near the electrode surface, which is sufficiently dry to prevent protonation of the reduced anionic states of the quinone. The effective hydrophobic blocking of the electrode support by the functionalized magnetic NPs is attributed to the partial carrying of toluene solvent by the hydrophobic nanoparticles [18]. The co-adsorbed toluene presumably forms a continued oil film that renders the interface into an organic phase microenvironment, which acts as a barrier for proton transfer. Thus, stepwise single-electron transfer to the monolayer-immobilized quinone units is demonstrated in the presence of hydrophobic magnetic NPs. Retraction of the hydrophobic magnetic NPs from the electrode surface to the upper toluene phase yields aqueous environment on the electrode surface, thus resulting in the aqueous-type of the quinone electrochemistry, Figure 1(C), curve (a). Magneto-induced cyclic attraction and retraction of the hydrophobic magnetic NPs to and from the quinone-functionalized electrode surface resulted in the reversible changes of the mechanism of the electrochemical process.

The control of the dielectric properties of electrode surfaces, and particularly of the surrounding of metal nanoparticles by means of the hydrophobic magnetic NPs allows the controlled switchable single-electron charging of the Au nanoparticles. The quantized (single electron) charging of metal NPs is a fundamental phenomenon. Single electron charging of metal nanoparticles at measurable potential steps, $\Delta \mathrm{E}$, is possible for small monodisperse metal nanoparticles provided that $\Delta \mathrm{E}>>\mathrm{k}_{\mathrm{B}} \mathrm{T}$, where $\Delta \mathrm{E}=\mathrm{e} / \mathrm{C}$ ( $\mathrm{C}$ is the capacitance of a single nanoparticle and e is the charge of electron). The single electron-charging phenomenon was observed for Au clusters protected by hydrophobic monolayers and in organic solvents of low dielectric constants [25-28]. The single-electron charging of metal nanoclusters on surfaces was accomplished by the addressing of single nanoparticles with a scanning tunneling microscopy tip [29], or by the electrical charging of nanoparticles arrays [30,31], consisting of small, monolayer-protected metal nanoparticles such as $\mathrm{Au}$ or Ag. Hydrophobic magnetic NPs were employed as a micro-heterogeneous environment for the single-electron charging of Au nanoparticles associated with a Au electrode surface. When the hydrophobic magnetic NPs are retracted from the Au NPs-functionalized electrode surface, the modified interface is in direct contact with the aqueous electrolyte solution, Figure 2(A), and the linear sweep voltammogram demonstrates the capacitance current characteristic to the aqueous environment, Figure 2(C), curve (a).

The hydrophobic magnetic NPs attracted to the electrode surface provide a microenvironment of low dielectric properties, and hence the low capacitance of the Au NPs allows their quantized charging, Figure 2(B). The linear sweep voltammogram corresponding to the quantized charging of the Au NPs is shown in Figure 2(C), curve (b). Five distinct charging waves, observed upon the negative potential sweep, correspond to the charging of the Au NPs by electrons. The waves are equally separated, $\Delta \mathrm{E}=$ 
$100 \pm 5 \mathrm{mV}$, and the charge associated with each wave is almost identical, $6.4 \pm 0.3 \mu \mathrm{C}$. Knowing the surface coverage of the Au NPs (determined by microgravimetric measurements), and the total charge associated with each quantized charging step, it was possible to estimate that ca. $1 \pm 0.05$ electrons are charging each Au NP in the array in each of the cathodic quantized steps. The removal of the hydrophobic magnetic NPs from the electrode surface resulted in the exposure of the Au NPs to the aqueous environment, Figure 2(A), thus resulting in the larger capacitance of the Au NPs. Under these conditions the quantum charging is not observed, Figure 2(C), curve (a). The reversible attraction and retraction of the hydrophobic magnetic NPs to and from the modified interface provides a method for the magneto-switchable single electron charging of a Au NPs array.

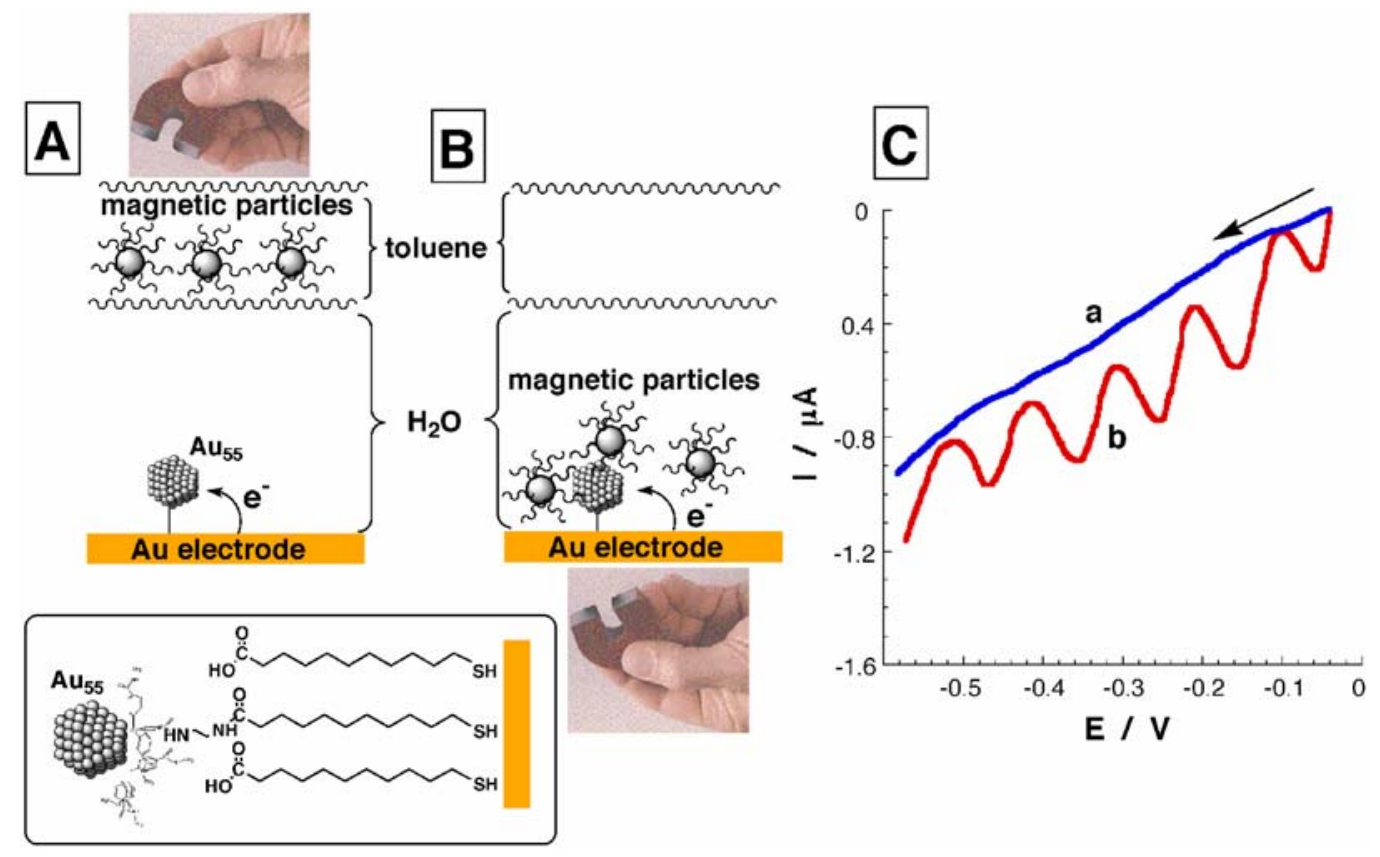

Figure 2. Magneto-controlled quantum charging of the Au NPs associated with a Au electrode in the presence of hydrophobic magnetic NPs and two-phase liquid solution: (A) Magnetic NPs are retracted from the electrode surface. (B) Magnetic NPs are attracted to the electrode surface. (C) Linear sweep voltammograms recorded in the presence of: a) Magnetic NPs retracted from the $\mathrm{Au}$ nanoparticles/thiol monolayer-functionalized electrodes. b) Magnetic NPs attracted to the Au nanoparticles/tiol monolayer-functionalized electrode. The data were obtained under $\mathrm{Ar}$ in a biphase system consisting of $0.1 \mathrm{M}$ phosphate buffer, $\mathrm{pH}$ 7.0, and toluene with the hydrophobic magnetic NPs, $0.5 \mathrm{~mL}, 1 \mathrm{mg} \mathrm{mL}^{-1}$. The effective electrode area is ca. $0.8 \mathrm{~cm}^{2}$. Potential scan rate $1 \mathrm{mV} \mathrm{s}^{-1}$. The arrow shows the direction of the potential sweep.

\section{Conclusions}

The present study has described a new method to control the mechanism of electrochemical reactions on modified electrode surfaces, namely to achieve quantized single-electron charging processes for molecular redox species (surface-confined quinone units) and metal NPs. We demonstrated that the mechanism of the electrochemical process of the quinone-modified electrode could be altered from an aqueous-type redox process to an organic-phase mechanism upon the attraction of the hydrophobic magnetic NPs to the electrode. The analysis of the results has implied 
that the assembly of the hydrophobic magnetic NPs on the electrode surface yields an isolating thin film that prevents the transport of protons/water molecules to the quinone-modified electrode surface. This property was attributed to the co-adsorption of toluene molecules to the hydrophobic magnetic NPs that results in an impermeable for $\mathrm{H}^{+} / \mathrm{H}_{2} \mathrm{O}$ thin film on the electrode. Also, a low dielectric constant of the hydrophobic microenvironment provided by the attracted magnetic NPs allows the quantized electron transfer to the Au NPs associated with the electrode surface. Reversible attraction and retraction of the hydrophobic magnetic NPs to and from the electrode surface allow the magnetocontrolled quantized and continues charging of the molecular redox units and metal NPs, respectively.

\section{References}

1. Shipway, A.N.; Katz, E.; Willner, I. Nanoparticle arrays on surfaces for electronic, optical and sensoric applications. ChemPhysChem., 2000, 1, 18.

2. Katz, E.; Willner, I. Integrated nanoparticle-biomolecule hybrid systems: Synthesis, properties and applications. Angew. Chem. Int. Ed., 2004, 43, 6042.

3. Zhao, X.J.; Tapec-Dytioco, R.; Wang, K.M.; Tan, W.H. Collection of trace amounts of DNA/mRNA molecules using genomagnetic nanocapturers. Anal. Chem., 2003, 75, 3476.

4. Wilson, R. Haptenylated mercaptodextran-coated gold nanoparticles for biomolecular assays. Chem. Commun., 2003, 108.

5. Lee, H.; Purdon, A.M.; Chu, V.; Westervelt, R.M. Controlled assembly of magnetic nanoparticles from magnetotactic bacteria using microelectromagnets arrays. Nano Lett., 2004, 4, 995.

6. Wang, J.; Kawde, A.-N. Magnetic-field stimulated DNA oxidation. Electrochem. Commun., 2002, 4, 349.

7. Wang, J.; Xu, D.K.; Polsky, R. Magnetically-induced solid-state electrochemical detection of DNA hybridization. J. Am. Chem. Soc., 2002, 124, 4208.

8. Li, J.S.; He, X.X.; Wu, Z.Y.; Wang, K.M.; Shen, G.L.; Yu, R.Q. Piezoelectric immunosensor based on magnetic nanoparticles with simple immobilization procedures. Anal. Chim. Acta, 2003, 481, 191.

9. Kourilov, V.; Steinitz, M. Magnetic-bead enzyme-linked immunosorbent assay verifies adsorption of ligand and epitope accessibility. Anal. Biochem., 2002, 311, 166.

10. Katz, E.; Sheeney-Haj-Ichia, L.; Willner, I. Magneto-switchable electrocatalytic and bioelectrocatalytic transformations. Chem. Eur. J., 2002, 8, 4138.

11. Willner, I.; Katz, E. Magnetic control of electrocatalytic and bioelectrocatalytic processes. Angew. Chem. Int. Ed., 2003, 42, 4576.

12. Katz, E.; Willner, I. Magneto-stimulated hydrodynamic control of electrocatalytic and bioelectrocatalytic processes. J. Am. Chem. Soc., 2002, 124, 10290.

13. Katz, E.; Willner, I. Enhancement of bioelectrocatalytic processes by the rotation of mediatorfunctionalized magnetic particles on electrode surfaces: Comparison with a rotating disk electrode. Electroanalysis, 2005, 17, 1616.

14. Patolsky, F.; Weizmann, Y.; Katz, E.; Willner, I. Magnetically amplified DNA assays (MADA): Sensing of viral DNA and single base mismatches using nucleic acid-modified magnetic particles. Angew. Chem. Int. Ed., 2003, 42, 2372.

15. Weizmann, Y.; Patolsky, F.; Katz, E.; Willner, I. Amplified DNA sensing and immunosensing by the rotation of functional magnetic particles. J. Am. Chem. Soc., 2003, 125, 3452. 
16. Patolsky, F.; Weizmann, Y.; Katz, E.; Willner, I. Amplified telomerase analysis by using rotating magnetic particles: The rapid and sensitive detection of cancer cells. ChemBioChem., 2004, 5, 943.

17. Katz, E.; Sheeney-Haj-Ichia, L.; Basnar, B.; Felner, I.; Willner, I. Magnetoswitchable controlled hydrophilicity/hydrophobicity of electrode surfaces using alkyl-chain-functionalized magnetic particles: Application for switchable electrochemistry. Langmuir, 2004, 20, 9714.

18. Katz, E.; Baron, R.; Willner, I. Magnetoswitchable electrochemistry gated by alkyl-chainfunctionalized magnetic nanoparticles: Controlling of diffusional and surface-confined electrochemical process. J. Am. Chem. Soc., 2005, 127, 4060.

19. Shen, L.F.; Laibinis, P.E.; Hatton, T.A. Bilayer surfactant stabilized magnetic fluids: Synthesis and interactions at interfaces. Langmuir, 1999, 15, 447.

20. Katz, E.; Schlereth, D.D.; Schmidt, H.-L. Electrochemical study of pyrroloquinoline quinone covalently immobilized as monolayer onto a cystamine modified gold electrode. J. Electroanal. Chem., 1994, 367, 59.

21. Katz, E.; Solov'ev, A.A. Chemical modification of platinum and gold electrodes by naphthoquinones using amines containing sulfhydryl or disulphide groups. J. Electroanal. Chem., 1990, 291, 171.

22. Berkovsky, B.M.; Medvedev, V.F.; Karkov, M.S. Magnetic Fluids: Engineering Applications, Oxford University Press, New York, 1993.

23. Rosensweig, R.R. Ferrohydrodynamics, Cambridge University Press, Cambridge, England, 1985.

24. Chambers, J.Q., In: The chemistry of the quinonoid compounds. Patai, S. (Ed.), Interscience, New York, 1974, p. 739.

25. Chen, S.W.; Ingram, R.S.; Hostetler, M.J.; Pietron, J.J.; Murray, R.W.; Schaaff, T.G.; Khoury, J.T.; Alvarez, M.M.; Whetten, R.L. Gold nanoelectrodes of varied size: Transition to molecule-like charging. Science, 1998, 280, 2098.

26. Chen, S.W.; Murray, R.W.; Feldberg, S.W. Quantized capacitance charging of monolayerprotected Au clusters. J. Phys. Chem. B, 1998, 102, 9898.

27. Chen, S. Nanoparticle assemblies: "Rectified" quantized charging in aqueous media. J. Am. Chem. Soc., 2000, 122, 7420.

28. Hicks J.F.; Templeton, A.C.; Chen, S.W.; Sheran, K.M.; Jasti, R.; Murray, R.W.; Debord, J.; Schaaf, T.G.; Whetten, R.L. The monolayer thickness dependence of quantized double-layer capacitances of monolayer-protected gold clusters. Anal. Chem., 1999, 71, 3703.

29. Andres, R.P.; Bein, T.; Dorogi, M.; Feng, S.; Henderson, J.I.; Kubiak, C.P.; Mahoney, W.; Osifchin, R.G.; Reifenberger, R. "Coulomb staircase" at room temperature in a self-assembled molecular nanostructures. Science, 1996, 272, 1323.

30. Markovich, G.; Leff, D.V.; Chung, S.-W.; Soyez, H.M.; Dunn, B.; Heath, J.R. Parallel fabrication and single-electron charging of devices based on ordered, two-dimensional phases of organically functionalized metal nanocrystals. Appl. Phys. Lett., 1997, 70, 3107.

31. Green, S.J.; Stokes, J.J.; Hostetler, M.J.; Pietron, J.; Murray, R.W. Three-dimensional monolayers: Nanometer-sized electrodes of alkanethiolate-stabilized gold cluster molecules. J. Phys. Chem. B, 1997, 101, 2663.

(C) 2006 by MDPI (http://www.mdpi.org). Reproduction is permitted for non-commercial purposes. 